\title{
Studies on the Nutrition and Growth Requirements of Mycoplasma gallisepticum
}

\author{
By CATHERINE G. FABRICANT, J. FABRICANT \\ AND P. J. VAN DEMARK \\ Division of Bacteriology, New York State College of Agriculture, and Department \\ of Avian Diseases, New York State Veterinary College, Cornell University, \\ Ithaca, New York, U.S.A.
}

(Received 29 October 1963)

\section{SUMMARY}

A complex tissue culture medium supplemented with swine serum and peptone supported optimal growth of Mycoplasma gallisepticum strain 293. Media lacking any of these components supported little or no growth. However, when $5^{\prime}$-monophosphate nucleotides replaced the peptone growth was supported. The minimal nucleotides necessary to support good growth were adenylic, cytidylic, guanylic and thymidylic acids. In some cases the addition of the four ribonucleotides and the four deoxyribonucleotides in place of peptone improved growth; the four ribonucleotides alone supported poor growth. Thymidylic acid seemed essential for growth, and uridylic acid appeared to be inhibitory.

The mixture of ribonucleosides and deoxyribonucleosides, but not of purines and pyrimidines, when substituted for the peptone, also supported good growth. The concentrations of nucleosides and nucleotides had a significant effect on growth. Although the nutritional factors of swine serum were not defined, the effects of different sera on growth were investigated. Rabbit, horse, turkey and swine serum supported optimum growth; human serum supported less, whereas PPLO serum fraction (Difco) or bovine serum supported poor growth. Dog serum did not support growth.

\section{INTRODUCTION}

The nutritional requirements of some Mycoplasma species have been reported by Edward (1947, 1953, 1954), Smith \& Morton (1951), Smith, Lecce \& Lynn (1954), Smith (1955), Chalquest \& Fabricant (1960), Rodwell (1960), Razin \& Knight $(1960 a, b)$, Razin (1962), and Razin \& Cohen (1963). It is apparent from these studies that the pathogenic as well as the saprophytic species have complex nutritional requirements. The main purpose of the present work was to evolve a defined medium which would support the growth of the avian pathogen Mycoplasma gallicepticum (Edward \& Kanarek, 1960) strain 293 and to determine its nutritional requirements. A partially defined medium described by Razin \& Knight (1960a), or modifications of it, did not support growth of the test organism. However, excellent growth was supported by a medium containing tissue culture (TC) medium 199 (Morgan, Morton \& Parker, 1950), swine serum and Bacto-peptone. This medium was selected as a basis for further work to replace the undefined components with defined ones. Media without the serum did not support 


\section{C. G. Fabricant, J. Fabricant and P. J. VanDemark}

growth. However, growth of the test organism did occur when the $5^{\prime}$-monophosphate ribonucleotides and deoxyribonucleotides, or the ribonucleosides and deoxyribonucleosides replaced the peptone component.

\section{METHODS}

Organism. Mycoplasma gallisepticum strain 293 was selected as the test organism. This strain was originally isolated from a field case of chronic respiratory disease in chickens by Dr J.R. E. Taylor in 1957; its morphological and physiological characteristics were described by Fabricant et al. (1959). Since isolation the organism has been maintained in continuous subculture on the same laboratory medium.

Media. Adler's overlay medium (Adler, Yamamoto \& Bankowski, 1954) was used for maintaining stock cultures and for growing the organism for inocula in the nutritional experiments.

Difco PPLO broth without crystal violet, enrichments or inhibitors was used as diluent in preparation of serial dilutions for colony counts. Difco PPLO agar enriched with $10 \%(\mathrm{v} / \mathrm{v})$ pooled swine serum, 1000 units penicillin $/ \mathrm{ml}$. and $0.05 \%$ $(\mathrm{w} / \mathrm{v})$ thallium acetate was used for colony counts. The pooled swine serum was obtained from a local slaughtering plant and sterilized by passage through a Seitz EK filter. All batches of serum were checked for ability to support growth of

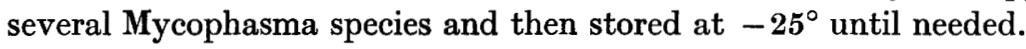

The medium used for the nutritional experiments was prepared from Difco TC medium 199 (based on the tissue culture medium of Morgan et al. 1950) liquid ten-fold concentrate. This medium was diluted to double concentration with sterile glass-distilled water, buffered with $0 \cdot 3 \%(\mathrm{w} / \mathrm{v}) \mathrm{K}_{2} \mathrm{HPO}_{4}$, rather than with sodium bicarbonate. After the addition of $10 \%(\mathrm{v} / \mathrm{v})$ pooled swine serum, and various supplements, the appropriate volume of distilled water was added to adjust the TC medium 199 to single concentration; when necessary, this was readjusted to

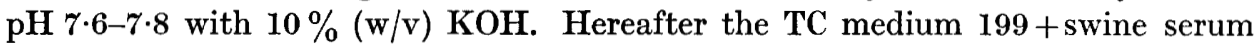
but without other supplements will be referred to as the basal medium. The control medium used in all nutritional experiments consisted of basal medium to which had been added $1 \%$ (w/v) Bacto-peptone.

Except for Adler's overlay medium all media, including all reagents and supplements, were prepared with glass-distilled water. Pyrex glassware was used in all experiments. Before use it was cleaned with chromic + sulphuric acid mixture and thoroughly rinsed with distilled water.

All nucleic acid derivatives and coenzymes were sterilized by passage through sintered glass filters. Undegraded DNA preparations were sterilized with a few drops of chloroform and then decanted from the chloroform. All other inorganic reagents and media used for colony counts were sterilized by autoclaving at $120^{\circ}$ for $15 \mathrm{~min}$.

Conditions of growth. Five ml. of basal medium with various supplements were dispensed in $13 \times 100 \mathrm{~mm}$. tubes with stainless steel closures. These were inoculated with $0.05 \mathrm{ml}$. of a $48 \mathrm{hr}$ stock culture. The cultures were incubated statically in air at $37^{\circ}$. Colony counts showed that the inoculum contained about $10^{6}$ organisms/ ml. medium.

Assessment of growth. The estimation of growth by turbidimetric methods or acid titration was unreliable under our experimental conditions; the basal medium 
was too turbid and there was no consistent correlation between acid production and colony counts. Therefore, growth was measured by the colony count technique described by Fabricant, VanDemark \& Fabricant (1962). All plates for colony counts were incubated in candle jars with excess moisture at $37^{\circ}$.

Chemicals. Undegraded DNA, the purines and pyrimidines adenine, cytosine, guanine, uracil and thymine, and the $5^{\prime}$-monophosphate deoxyribonucleotides, deoxyadenylic acid disodium salt. $3 \mathrm{H}_{2} \mathrm{O}$ (dAMP), deoxycytidylic acid. $2 \mathrm{H}_{2} \mathrm{O}$ (dCMP), deoxyguanulate. $\mathrm{NH}_{4}$ (dGMP), and thymidylic acid sodium salt (TMP) were products of the California Corporation for Biochemical Research (Los Angeles, California). The 5'-monophosphate ribonucleotides muscle adenylic acid (AMP), guanylic acid sodium salt (GMP), cytidylic acid sodium salt (CMP), and uridylic acid disodium salt (UMP) were products of the Pabst Laboratories (Milwaukee, Wisconsin) or the Sigma Chemical Company (St Louis, Missouri). The 5'-triphosphate nucleotides of adenosine, disodium salt (ATP), cytidine, sodium salt (CTP), guanosine, sodium salt (GTP), and uridine, sodium salt (UTP) were also products of the Pabst Laboratories. The ribonucleosides adenosine, cytidine, guanosine, uridine, and the deoxyribonucleosides, deoxyadenosine, deoxycytidine.HCl, deoxyguanosine, and thymidine were products of the Nutritional Biochemical Corporation (Cleveland, Ohio).

\section{RESULTS}

The partially defined medium described by Razin \& Knight (1960a) for a strain of Mycoplasma laidlawii did not permit survival of Mycoplasma gallisepticum strain 293. Attempts to improve this medium by addition of PPLO serum fraction (Difco), $10 \%(\mathrm{v} / \mathrm{v})$ swine serum, increased amounts of glucose, or biotin and folic acid also failed and further work with it was abandoned.

Initial attempts to grow the test organism in TC medium 199 with or without $10 \%(\mathrm{v} / \mathrm{v})$ swine serum also failed. Since this medium changed from $\mathrm{pH} 7 \cdot 6$ to $8 \cdot 9$ within $3 \mathrm{hr}$ of static incubation at $37^{\circ}$, the medium was subsequently buffered with $0.3 \%(\mathrm{w} / \mathrm{v}) \mathrm{K}_{2} \mathrm{HPO}_{4}$ rather than with sodium bicarbonate as recommended. Inocula of the test organism survived better in this medium, without serum and buffered with $\mathrm{K}_{2} \mathrm{HPO}_{4}$, than in media buffered with sodium bicarbonate or tris buffer ( $\mathrm{pH} 7-9$; Sigma).

However, minimal growth of strain 293 occurred when the TC medium 199 was supplemented with $10 \%(\mathrm{v} / \mathrm{v})$ swine serum (basal medium). Addition of $0 \cdot 1-0.4 \%$ $(\mathrm{w} / \mathrm{v})$ of glucose to this basal medium did not improve growth.

Other studies indicated that the basal medium lacked some essential nutritional factor, or factors, which could be supplied by Difco PPLO broth (without crystal violet). The factor, or factors, could be supplied equally by $1 \%(\mathrm{w} / \mathrm{v})$ Bacto-peptone, one of the PPLO broth ingredients (see Table 1), and to a lesser degree by beef heart for infusion, the other PPLO broth ingredient.

The basal medium supplemented with $1 \%(\mathrm{w} / \mathrm{v})$ Bacto-peptone was used as the control medium in all later experiments. Optimal growth of strain 293 was obtained only in the control medium. No growth occurred in media when either TC medium 199 or serum were omitted.

Optimal growth of the test organism also occurred in media where the concentration of peptone (in the control medium) was altered from 0.4 to $4 \%(\mathrm{w} / \mathrm{v})$. 


\section{C. G. Fabricant, J. Fabricant and P. J. VanDemark}

Concentration of $6,0 \cdot 2$ and $0 \cdot 1 \%$ peptone yielded less growth, while concentrations above $6 \%$ were inhibitory.

Essentially the same amount of growth occurred in media where rabbit, horse, or turkey serum was substituted for one lot of swine serum in the control medium (see Table 2). Human serum supported moderate growth, Difco PPLO serum fraction or bovine serum supported poor growth, while dog serum did not support growth. Other experiments indicated that some batches of swine serum were superior to horse serum in supporting growth of strain 293 in the control medium. Neither tryptic digests of casein nor peptone prepared according to the method of Roberts \& Snell (1946), when substituted for the peptone of the control medium, allowed growth of strain 293.

Table 1. The growth of Mycoplasma gallisepticum strain 293 in TC medium $199+$ swine serum (basal medium) supplemented with Difco PPLO broth, Bacto-peptone, or beef heart for infusion (Difco)

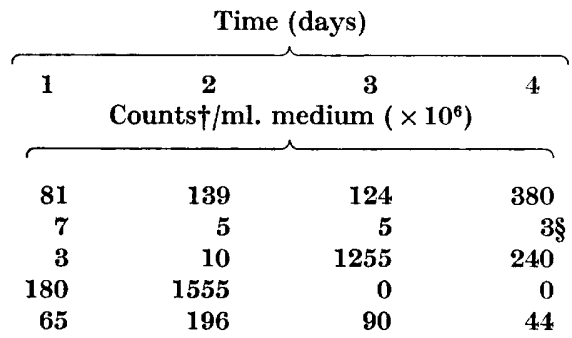

PPLO broth + swine serum $\ddagger$

Basal medium

Basal medium + PPLO broth

Basal medium $+1 \%(w / v)$ peptone

Basal medium $+5 \%(w / v)$ beef heart

65

196

90

for infusion

* Since the inoculum did not survive in any of the above mixtures of medium components without serum, these counts are not given in the Table.

$\dagger$ The inoculum contained $4 \times 10^{6}$ viable particles $/ \mathrm{ml}$. medium. All counts have been rounded off to the nearest $10^{6}$ figure.

$\ddagger$ Swine serum was added in $10 \%(v / v)$ to all media.

$\S$ Marked decrease in all counts after initial growth was due to acid production.

Table 2. The growth of Mycoplasma gallisepticum strain 293 in TC medium 199 supplemented with peptone and various pooled sera or PPLO serum fraction (Difco)

\begin{tabular}{|c|c|c|c|c|}
\hline & \multicolumn{4}{|c|}{ Time (days) } \\
\hline & 1 & 2 & $\mathbf{3}$ & 4 \\
\hline & \multicolumn{4}{|c|}{ Counts $\uparrow / \mathrm{ml}$. medium $\left(\times 10^{6}\right)$} \\
\hline Rabbit serum & 295 & 855 & $3+$ & $\mathbf{0}$ \\
\hline Horse serum & 795 & $\mathbf{5 0 0}$ & $<1$ & 0 \\
\hline Swine serum & 705 & $\mathbf{5 3 0}$ & $<1$ & 0 \\
\hline Turkey serum & 605 & $<1$ & $\mathbf{0}$ & 0 \\
\hline Human serum & 105 & $\mathbf{2 3 0}$ & $<1$ & $<1$ \\
\hline Bovine serum & 22 & 6 & 10 & 6 \\
\hline PPLO serum fraction & 9 & 6 & 8 & 7 \\
\hline Dog serum & $<1$ & $<1$ & $<1$ & $<1$ \\
\hline
\end{tabular}

* TC medium 199 was buffered with $0 \cdot 3 \%(w / v) \mathrm{K}_{2} \mathrm{HPO}_{4}$ supplemented with $1 \%$ (w/v) peptone and $10 \%(v / v)$ of the various sera or $1 \%(v / v)$ of Difco PPLO serum fraction.

$\dagger$ The inoculum contained $7 \times 10^{6}$ viable particles $/ \mathrm{ml}$. medium; all counts have been rounded off to the nearest $10^{6}$ figure.

$\ddagger$ Marked decrease in counts after initial growth was due to acid production. 
Further work involved attempts to replace the serum and peptone of the control medium with chemically defined factors. However, none of the factors tested were capable of replacing the serum and only data concerning replacement of the peptone will be included here.

Media in which the peptone of the control medium was replaced by the 5 -monophosphate ribonucleotides or ribonucleosides, where these were added in the same concentration as the free purine and pyrimidine bases already present in TC medium 199, supported slight growth of the test organism. The addition of coenzyme A, diphosphopyridine nucleotide (DPN), flavin adenine dinucleotide (FAD) and $\alpha$-lipoic acid (added in $0.25,0.7,0.1,0.001 \mathrm{mg} . / 100 \mathrm{ml}$. medium, respectively) to these media did not stimulate growth. Nor did the addition of reduced glutathione and L-cysteine. $\mathrm{HCl} . \mathrm{H}_{2} \mathrm{O}(100 \mathrm{mg}$. each/100 ml. medium) improve the growth in these media.

Table 3. The influence of ribonucleotides $A M P, C M P, G M P$, deoxyribonucleotide $T M P$, and DNA on the growth of Mycoplasma gallisepticum strain 293 in the basal medium

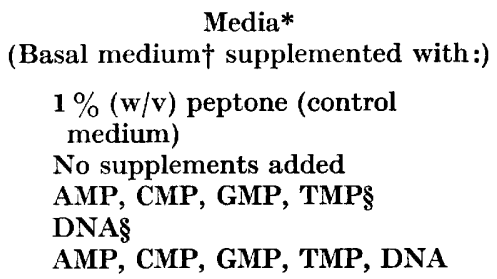

\begin{tabular}{|c|c|c|c|c|}
\hline \multicolumn{5}{|c|}{ Time (days) } \\
\hline $\mathbf{1}$ & 2 & 3 & 4 & 5 \\
\hline \multicolumn{5}{|c|}{ Counts $\$ / \mathrm{ml}$. medium $\left(\times 10^{6}\right)$} \\
\hline 320 & 600 & 34 & $\mathbf{2 4} \|$ & - \\
\hline 71 & 3 & 2 & 4 & - \\
\hline 4 & 8 & 92 & 48 & - \\
\hline 1 & 30 & 53 & 140 & 29 \\
\hline 71 & 8 & 75 & 350 & 42 \\
\hline
\end{tabular}

* Media combinations without serum have been omitted since they did not support growth.

$\dagger$ Basal medium contained TC medium 199 buffered with $0 \cdot 3 \%(w / v) \mathrm{K}_{2} \mathrm{HPO}_{4}$ and $10 \%(\mathrm{v} / \mathrm{v})$ swine serum.

\$ The inoculum contained $13 \times 10^{6}$ viable particles $/ \mathrm{ml}$. medium. All counts have been rounded off to the nearest $10^{6}$ figure.

$\S$ Nucleotides and undegraded DNA each added in $1 \mathrm{mg} . / 100 \mathrm{ml}$. medium.

\| Marked decrease in all counts after initial growth was due to acid production.

However, as shown in Table 3, growth was stimulated when the $\mathbf{5}^{\prime}$-monophosphate ribonucleotides AMP, CMP, GMP and the same isomer of deoxyribonucleotide TMP were added in $1 \mathrm{mg} . / 100 \mathrm{ml}$. basal medium (nucleotides replacing the peptone of the control medium). As further shown in Table 3, growth was markedly stimulated when DNA (1 mg./100 ml. medium) was added to the basal medium supplemented with these four nucleotides.

In view of the growth of strain 293 in basal medium + DNA only, and the stimulation of growth by DNA in the medium containing the nucleotides, AMP, CMP, GMP, and TMP, the four $5^{\prime}$-monophosphate deoxyribonucleotides were substituted for the DNA to determine their comparative effects on growth. Table 4 shows that the basal medium supplemented with the four ribonucleotides alone supported the least growth. The preparation of DNA or the nucleotides AMP, CMP, GMP, UMP and TMP supplementing the basal medium supported the same amount of growth. Better growth was supported in the medium containing AMP, CMP, GMP, and TMP, while slightly less growth occurred in the medium containing both the 
ribonucleotides and the deoxyribonucleotides. The medium containing the deoxyribonucleotides alone supported growth intermediate between the last two media named.

As also shown in Table 4, the addition of the $5^{\prime}$-triphosphate nucleotide ATP to the medium containing the eight monophosphate nucleotides, further stimulated growth of strain 293. The addition of the four triphosphate nucleotides ATP, CTP, GTP and UTP to this medium stimulated only slightly more growth than ATP alone. In this experiment the triphosphate nucleotides were prepared and stored frozen for 1 week before use. In other experiments freshly prepared ATP did not show the same growth stimulation.

Table 4. The influence of 5'-monophospho-ribonucleotides, deoxyribonucleotides, DNA and triphosphonucleotides on the growth of Mycoplasma gallisepticum strain 293 in the basal medium

\begin{tabular}{|c|c|c|c|}
\hline \multicolumn{4}{|c|}{ Time (days) } \\
\hline 1 & 2 & $\mathbf{3}$ & 4 \\
\hline \multicolumn{4}{|c|}{ Counts*/ml. medium $\left(\times 10^{6}\right)$} \\
\hline 190 & 640 & 480 & 54 \\
\hline 6 & 18 & 18 & 2 \\
\hline 10 & 26 & 47 & $<1$ \\
\hline 28 & 56 & 77 & $<1$ \\
\hline 8 & 101 & 8 & $<1$ \\
\hline 8 & 22 & 48 & 39 \\
\hline 18 & 56 & 60 & $<1$ \\
\hline 12 & 69 & 115 & $<1$ \\
\hline 9 & 67 & 50 & $<1$ \\
\hline 23 & 190 & 4 & $<1$ \\
\hline 12 & 215 & 10 & $<1$ \\
\hline
\end{tabular}

Media (basal medium $\uparrow$ supplemented with:)

I. $1 \%(\mathrm{w} / \mathrm{v})$ peptone (control medium)

II. AMP, CMP, GMP, UMP $\$ 8$

III. AMP, CMP, GMP, UMP, TMP

IV. AMP, CMP, GMP, TMP

V. AMP, CMP, GMP, TMP, DNA

VI. DNA

VII. dAMP, dCMP, dGMP, TMP

VIII. AMP, CMP, GMP, UMP + DNA

IX. AMP, CMP, GMP, UMP, dAMP, dCMP, dGMP, TMP

X. Medium IX with ATP+

XI. Medium IX with ATP, CTP, GTP, UTP

* Inoculum contained $13 \times 10^{6}$ organisms per ml. of medium. All counts have been rounded off to the nearest $10^{6}$ figure.

$\dagger$ Basal medium contained TC medium 199 buffered with $0 \cdot 3 \%(w / v) \mathrm{K}_{2} \mathrm{HPO}_{4}$ and $10 \%(\mathrm{v} / \mathrm{v})$ swine serum.

$\mp$ All ribo- and deoxyribonucleotides and DNA were added in $1 \mathrm{mg}$. each/100 ml. of medium. The triphosphonucleotides were added in $0 \cdot 1 \mathrm{mg}$. each $/ 100 \mathrm{ml}$. of medium.

§ The 5'-monophosphoribonucleotides.

i| The 5'-monophosphodeoxyribonucleotides.

Tarked drop in all counts after initial growth was due to acid production.

Further effects on the growth of strain 293 in the basal medium supplemented with various mixtures or concentrations of nucleotides are illustrated in Table 5. The results indicate that the concentration of the eight monophospate nucleotides significantly influenced the amount of growth. Growth was essentially doubled when the concentration of these nucleotides was halved, whereas doubling their concentration supported approximately half as much growth. In this experiment the medium containing the eight monophosphate nucleotides supported slightly more growth than that containing only AMP, CMP, GMP and TMP. The omission of UMP from the medium containing the seven other nucleotides yielded more growth than the medium including UMP. In this same experiment, a week-old preparation of ATP had a stimulatory effect on growth in the medium containing the eight monophosphate nucleotides, whereas adenosine diphosphate (ADP) seemed to have an inhibitory effect. When ATP and ADP were added together, the resulting growth was essentially the same as that in the medium where ATP was added alone. 
Table 6 compares the effects of growth of strain 293 in the basal medium supplemented with the eight monophosphate nucleotides, the corresponding eight nucleosides, or the free purine and pyrimidine bases. The concentrations of the nucleosides

Table 5. The influence of various combinations and concentrations of 5'-monophosphonucleotides and the adenine diphospho- and triphosphonucleotides ADP, ATP) on grozoth of Mycoplasma gallisepticum strain 293 in the basal medium

Media (basal medium* supplemented with:)

I. $1 \%(w / v)$ peptone (control medium)

II. AMP, CMP, GMP, UMP, dAMP, dCMP, dGMP, TMP +

III. Medium II without UMP

IV. AMP, CMP, GMP, TMP

V. Medium II with nucleotides at $1 / 2$ concentration

VI. Medium II with nucleotides at $2 \times$ concentration

VII. Medium II plus ATP\$

VIII. Medium II plus ADP\$

IX. Medium II plus ADP and ATP

\begin{tabular}{|c|c|c|}
\hline \multicolumn{3}{|c|}{ Time (days) } \\
\hline 1 & 2 & 3 \\
\hline \multicolumn{3}{|c|}{ Counts $\uparrow / \mathrm{ml}$. medium $\left(\times 10^{6}\right)$} \\
\hline 435 & 745 & 80 \\
\hline 15 & 66 & 7 \\
\hline 38 & 94 & 3 \\
\hline 13 & 51 & 43 \\
\hline 32 & 114 & 2 \\
\hline 28 & 37 & $\overline{1}$ \\
\hline 22 & 102 & 9 \\
\hline 23 & 18 & $\mathbf{2 3}$ \\
\hline 33 & 99 & 29 \\
\hline
\end{tabular}

* Basal medium contained TC medium 199 buffered with $0 \cdot 3 \%$ (w/v) $\mathrm{K}_{2} \mathrm{HPO}_{4}$ and $10 \%$ (v/v) swine serum.

$\dagger$ The inoculum contained $12 \times 10^{6}$ viable particles $/ \mathrm{ml}$. of medium. All counts have been rounded off to the nearest $10^{6}$ figure.

\$ The first four listed are the $5^{\prime}$-ribonucleotides, the others are the $5^{\prime}$-deoxyribonucleotides; each was added in $1 \mathrm{mg} . / 100 \mathrm{ml}$. of medium. (Monophosphate nucleotides).

$\S$ ADP and ATP were each added in $0.1 \mathrm{mg} .100 \mathrm{ml}$. of medium.

II Slight increase in growth at 3 days was followed in four days by a drop to less than $1 \times 10^{6}$. Marked decrease in all counts after initial growth was due to acid production.

and the purine and pyrimidine bases added were calculated to approximate the concentrations of the nucleotides, each of which was added at $1 \mathrm{mg} . / 100 \mathrm{ml}$. medium. Growth was slightly greater in the medium containing nucleosides than that containing nucleotides. Although growth was increased in both media when the concentration of nucleosides or nucleotides was halved, considerably more growth resulted in the medium with the lower concentration of nucleosides. However, it was noted in the course of colony counts, that subcultures to plating agar from media containing nucleosides resulted in a large number of atypical rough colonies. Atypical colonies were rarely, if ever, noted in subcultures during the growth phase of strain 293 from media containing nucleotides. The substitution of purines and pyrimidines for the peptone of the control medium resulted in minimal growth. It was further noted that all colonial growth on subcultures to plating agar was extremely rough and atypical.

\section{DISCUSSION}

Since the tissue culture medium (TC medium 199) of Morgan et al. (1950) contains a large complement of amino acids, vitamins, purines, pyrimidines, inorganic salts and cholesterol, it was selected as the base medium from which to evolve a defined medium capable of supporting growth of Mycoplasma gallisepticum strain 293. 


\section{C. G. Fabricant, J. Fabricant and P. J. VanDemark}

Table 6. The relative influence of the 5'-monophospho-, ribo- and deoxyribonucleotides, the corresponding nucleosides, or the free purine and pyrimidine bases on growth of Mycoplasma gallisepticum strain 293 in the basal medium

$\begin{array}{rrrr}\overbrace{1}^{4} \begin{array}{c}2 \\ \text { Counts } \dagger / \text { ml. medium }\left(\times 10^{6}\right)\end{array} & 4 \\ 630 & 705 & 120 \S & - \\ 59 & 81 & 12 & <1 \\ 53 & 105 & 62 & <1 \\ 54 & 100 & 6 & <1 \\ 86 & 167 & 8 & <1 \\ 5 & 12 & 12 & <1 \\ 8 & 9 & 12 & 2\end{array}$

Media (basal medium* supplemented with:)

I. $1 \%(w / v)$ peptone (control medium)

II. AMP, CMP, GMP, UMP, dAMP, dCMP, dGMP, TMP

III. Medium II with nucleotides at $1 / 2$ concentration

IV. Nucleosides $\ddagger$

V. Medium IV with nucleosides at $1 / 2$ concentration

VI. Purines and pyrimidines $\ddagger$

VII. Medium VI with purines and pyrimidines at $1 / 2$ concentration

* Basal medium contained TC medium 199 buffered with $0.3 \%(w / v) \mathrm{K}_{2} \mathrm{HPO}_{4}$ and $10 \%(\mathrm{v} / \mathrm{v})$ swine serum.

$\uparrow$ The inoculum contained $10 \times 10^{6} \mathrm{organisms} / \mathrm{ml}$. of medium. All counts have been rounded off to the nearest $10^{6}$ figure.

$¥$ The first four listed are the ribonucleotides, the others are the deoxyribonucleotides; each was added in $1 \mathrm{mg} . / 100 \mathrm{ml}$. of medium. Calculated equivalent concentrations of the corresponding nucleosides (adenosine, cytidine, guanosine, deoxyadenosine, deoxycytidine, deoxyguanosine, and thymidine) and purines and pyrimidines (adenine, cytosine, guanine, uracil and thymine) were added to media IV and VI respectively.

$\S$ Marked drop in all counts after initial growth was due to acid production.

This medium, however, was found to lack essential nutrients which could be supplied by adding serum + Bacto-peptone. Optimal growth of strain 293 occurred only in media containing the TC medium 199 + serum + peptone, omission of any of these three components resulted in little or no growth. It is likely that all the components of TC medium 199 are not essential for growth of strain 293. The present work and the report of Razin (1962) suggest that the purines and pyrimidines might be eliminated. It is also possible that the essential ingredients of this medium are not at optimal concentrations. However, it was felt that until defined reagents were found capable of adequately replacing the serum and peptone parts of the control medium, it would be unprofitable to determine the effects of the individual components of TC medium 199 on growth of strain 293.

The reports of Edward (1954), Chalquest \& Fabricant (1960) and Razin \& Knight $(1960 b)$ suggested that nucleic acid precursors or other known protein derivatives might replace either or both undefined components (serum, peptone) of the control medium. Professor R. W. Holley suggested (personal communication) that the preformed ribonucleosides or the $\mathbf{5}^{\prime}$-monophosphate ribonucleotides of adenine, cytosine, guanine and uracil, rather than purines and pyrimidines might be essential for growth of organisms with a limited content of enzymes. Therefore these ribonucleosides or ribonucleotides were substituted for either or both serum and peptone of the control medium. Initial studies indicated that the ribonucleotides, rather than the ribonucleosides, would partially replace the peptone, but not the serum, in supporting growth of strain 293. However, better growth resulted when 
peptone was replaced by the ribonucleotides AMP, CMP, GMP and the deoxyribonucleotide ( 5 '-monophosphate isomer) thymidylic acid (TMP). It was found that the best growth occurred when each of these nucleotides was added in the same concentration. These findings parallel those of Razin (1962) in which he reported that mixtures of the $5^{\prime}$-monophosphate ribonucleotides were active in growth promotion of Mycoplasma laidlawii strain A.

Comparison of growth in media where the $5^{\prime}$-monophosphate nucleotides of RNA, or DNA or mixtures of these were substituted for the peptone (each nucleotide added to $1 \mathrm{mg} . / 100 \mathrm{ml}$. medium) showed that the four RNA nucleotides, AMP, CMP, GMP and UMP, gave the poorest growth. The four DNA nucleotides, dAMP, dCMP, dGMP and TMP, or the RNA and DNA nucleotides together gave about four times as much growth as the RNA nucleotides alone. However better growth was given by the nucleotides AMP, CMP, GMP and TMP than by the four DNA nucleotides only. The experimental evidence indicates that in these media TMP may be essential for growth while UMP may be somewhat inhibitory.

In one experiment when undegraded DNA replaced the peptone in the control medium there was more growth than in the media containing nucleotides. However, in a later experiment various mixtures of nucleotides (supplementing the basal medium) were superior to undegraded DNA. Further work may resolve this discrepancy. The effect of ATP on growth of strain 293 in basal medium supplemented with nucleotides also needs clarification. Week-old preparations of ATP had a stimulatory effect on growth, while fresh preparations did not. The difference did not seem to be due to the possible breakdown of ATP to ADP, since ADP seemed to have an inhibitory effect on growth in one trial.

Comparison of growth of Mycoplasma gallisepticum strain 293 in the basal medium supplemented by the RNA and DNA nucleosides, 5'-monophosphate nucleotides or purines and pyrimidines, parallels in part the results reported by Razin (1962) for $M$. laidlawii strain A. He found that purines and pyrimidines would not stimulate growth of $\boldsymbol{M}$. laidlarwii strain $\mathbf{A}$ in his partially defined medium. The minimal growth of strain 293 in our basal medium supplemented with purines and pyrimidines was probably due to the basal medium alone. Furthermore, the markedly rough and atypical colonial growth from these media subcultured on plating agar, suggests that purines and pyrimidines may disrupt typical colonial development of strain 293. Growth of $M$. gallisepticum strain 293 was better in media containing nucleosides than in those containing nucleotides; similar results were reported by Razin (1962) for $M$. laidlawii. However, we noted that a large proportion of colonies plated from media with nucleosides was rough and atypical. Atypical colonies were not noted during the growth phase of strain 293 in media containing nucleotides. Since the only difference between the nucleosides and nucleotides was the phosphate linkage of the latter it is possible that the preformed nucleotides may be better utilized by strain 293 for subsequent typical colonial growth.

Further work is needed to establish the optimum concentrations and mixtures of nucleosides or nucleotides for growth of strain 293 in the basal medium. The concentration of $0.5 \mathrm{mg}$. of each nucleoside or nucleotide/100 ml. medium supported more growth than double this concentration. Further increase in concentration of nucleotides (and possibly nucleosides) was inhibitory. 


\section{C. G. Fabricant, J. Fabricant and P. J. VanDemark}

We found that the minimal nucleotides which supported good growth of Mycoplasma gallisepticum strain 293 were adenylic, cytidylic, guanylic and thymidylic acids ( $5^{\prime}$-monophosphates). Future work may establish that the minimal nucleoside requirements of strains 293 may be adenosine, cytidine, guanosine and thymidine. This would parallel the minimal nucleoside requirements for growth of $M$. laidlawii strain A as reported by Razin (1962) in his partially defined medium.

We wish to thank Professor R. W. Holley (U.S. Plant, Soil and Nutrition Laboratory at Cornell University, Ithaca, New York) for his assistance and guidance in the work with nucleosides and nucleotides. We also thank Professors S. A. Zahler, R. E. MacDonald, H. B. Naylor and E. A. Delwiche of the Laboratory of Bacteriology at Cornell for helpful advice and the loan of some chemicals and equipment. This work was supported in part by Grant No. AI-04318-02 from the U.S. National Institutes of Health.

\section{REFERENCES}

Adler, H. E., Yamamoto, R. \& Bankowski, R. A. (1954). A preliminary report of efficiency of various mediums for isolation of pleuropneumonia-like organisms from exudates of birds with chronic respiratory disease. Amer. J. vet. Res. 15, 463.

Chalquest, R. R. \& Fabricant, J. (1960). Pleuropneumonia-like organisms associated with synovitis in fowl. Avian Dis. 4, 515.

EDWARD, D. G. FF. (1947). A selective medium for pleuropneumonia-like organisms. J. gen. Microbiol. 1, 237.

EDWARD, D. G. FF. (1953). A difference in growth requirements between bacteria in the L-phase and organisms of the pleuropneumonia group. J. gen. Microbiol. 8, 256.

EDWARD, D. G. FF. (1954). The pleuropneumonia group of organisms: A review together with some new observations. J. gen. Microbiol. 10, $2 \%$.

EDWard, D. G. FF. \& KanareK, A. D. (1960). Organisms of the pleuropneumonia group of avian origin: their classification into species. Ann. N.Y. Acad. Sci. 79, 696.

Fabricant, J., Levine, P. P., Calnek, B. W., Adler, H. E. \& Berg, J. (1959). Studies of egg transmission of PPLO in chickens. Avian Dis. 3, 197.

Fabricant, C. G., VanDemark, P. J. \& Fabricant, J. (1962). The effect of atmospheric environment upon growth of Mycoplasma gallisepticum. Avian Dis. 6, 328.

Morgan, J. F., Morton, H. J. \& Parker, R. C. (1950). Animal cells in tissue culture in initial studies on a synthetic medium. Proc. Soc. exp. Biol., N.Y. 73, 1.

Razin, S. (1962). Nucleic acid precursor requirements of Mycoplasma laidlarwii. J. gen. Microbiol. 28, 243.

Razin, S. \& Cohen, A. (1963). Nutritional requirements and metabolism of Mycoplasma laidlawii. J. gen. Microbiol. 30, 141.

Razin, S. \& Knight, B. C. J. G. (1960a). A partially defined medium for the growth of Mycoplasma. J. gen. Microbiol. 22, 492.

RaziN, S. \& Knight, B. C. J. G. (1960b). The effects of ribonucleic acid and deoxyribonucleic acid on growth of Mycoplasma. J. gen. Microbiol. 22, 504.

Roberts, E. C. \& SNell, E. E. (1946). An improved medium for microbiological assays with Lactobacillus casei. J. biol. Chem. 163, 499.

RoDwell, A. W. (1960). The nutrition and metabolism of Mycoplasma mycoides var. mycoides. Ann. N.Y. Acad. Sci. 79, 499.

Sмiтн, P. F. (1955). Synthetic media for pleuropneumonia-like organisms. Proc. Soc. exp. Biol. N.Y. 88, 628.

Smith, P. F., LeCCE, J. C. \& LynN, R. J. (1954). A lipoprotein as a growth factor for certain pleuropneumonia-like organisms. $J$. Bact. 68, 627 .

Smith, P. F. \& Morton, H. E. (1951). A separation and characterization of the growth factor in serum and ascitic fluid which is required by certain pleuropneumonia-like organisms. J. Bact. 61, 395. 